ÉGYPTE

monde arabe

\section{Égypte/Monde arabe}

10 | 2013

Les élections de la révolution (2011-2012)

\title{
Les femmes algériennes au Parlement : la question des quotas à l'épreuve des réformes politiques
}

\section{Belkacem Benzenine}

\section{(2) OpenEdition}

1 Journals

\section{Édition électronique}

URL : https://journals.openedition.org/ema/3196

DOI : 10.4000/ema.3196

ISSN : 2090-7273

\section{Éditeur}

CEDEJ - Centre d'études et de documentation économiques juridiques et sociales

\section{Édition imprimée}

ISBN : 978-2-905838-81-0

ISSN : $1110-5097$

\section{Référence électronique}

Belkacem Benzenine, « Les femmes algériennes au Parlement : la question des quotas à l'épreuve des réformes politiques ", Égypte/Monde arabe [En ligne], 10 | 2013, mis en ligne le 11 février 2014, consulté le 07 juillet 2022. URL : http://journals.openedition.org/ema/3196 ; DOI : https://doi.org/ 10.4000/ema.3196

Ce document a été généré automatiquement le 7 juillet 2022.

Tous droits réservés 


\title{
Les femmes algériennes au Parlement : la question des quotas à l'épreuve des réformes politiques*
}

\author{
Belkacem Benzenine
}

1 Le 10 mai 2012 marque une date importante dans l'histoire des femmes en Algérie. Les élections législatives organisées en ce jour ont en effet permis à 146 femmes d'accéder à l'Assemblée populaire nationale (APN, chambre basse du Parlement ${ }^{1}$ ), soit un taux de $31,6 \%$, alors qu'il n'était que de 7,7\% auparavant, puisque seulement 29 femmes ont siégé dans la précédente législature (2007-2012). Ainsi, avec les résultats des élections de mai 2010, l'Algérie occupe le premier rang dans le monde arabe et le $28^{\text {ème }}$ rang à l'échelle internationale en matière de la représentation des femmes aux parlements nationaux ${ }^{2}$.

2 Cette avancée est la conséquence de la loi organique du 12 janvier 2012 adoptée en application de l'article 31 bis de la Constitution, telle qu'amendée en 2008. Cet article stipule que «l'État œuvre à la promotion des droits politiques de la femme en augmentant ses chances d'accès à la représentation dans les assemblées élues ». Il s'agit d'une reconnaissance constitutionnelle qui consacre le renforcement de la participation des femmes aux assemblées élues, et contribue à la promotion de l'égalité entre les sexes. La présence des femmes sur les listes électorales a été pour la première fois un enjeu central dans ces élections législatives, un enjeu qui se situe à la confluence des attentes des femmes et de la volonté du pouvoir politique de promouvoir la représentation politique féminine. L'idée sous-jacente est qu'une forte présence des femmes dans les assemblées élues est à la fois la clé de leur autonomie et l'accomplissement du rôle de l'état dans la consolidation du principe d'égalité.

3 Notre contribution vise à montrer comment se pose la question de la représentation politique des femmes algériennes en s'intéressant particulièrement à la loi organique sur les quotas adoptée en 2012. Elle s'interroge également sur les éventuelles conséquences de la forte présence féminine au Parlement, sur la vie politique et sur la condition des femmes en Algérie. L'adoption d'une loi imposant des quotas pour les 
femmes est-elle la consécration d'un féminisme d'état? Une telle loi était-elle nécessaire ? En quoi cette loi pouvait-elle contribuer à optimiser les compétences des femmes? Comment les partis politiques ont-ils réagi? Comment les mouvements féminins ont-ils pesé dans les débats relatifs à cette loi ? Ces questions se rapportent à l'expérience algérienne en matière de quota de femmes, et aux problèmes soulevés par la classe politique et les femmes elles-mêmes quant à l'application de cette loi.

\section{Les femmes et la politique dans le contexte des élections législatives de 2012}

4 Les élections législatives de mai 2012 se sont déroulées dans un contexte marqué par de fortes contestations sociales. Sur le plan politique cependant, l'Algérie n'a pas subi les pleins effets du Printemps arabe. Le pouvoir en place, surtout depuis l'arrivée de Bouteflika à la présidence de la République en 1999, a relativement réussi à faire face au mouvement de protestation politique. L'organisation des élections dans les délais constitutionnels a représenté pour le pouvoir une opportunité de se doter de la légitimité nécessaire (étant entendu que le régime algérien est «un autoritarisme électoral au sein duquel la pratique politique se caractérise par son aspect limité $\left.»^{3}\right)$. En outre, les élections législatives de 2012 ont été pour le régime une occasion de faire montre de sa volonté de réaliser le changement ambitionné par le peuple, et ce à travers les urnes. Dans un processus dit de réformes politiques, décidées par le pouvoir et s'inscrivant dans une tentative d'apaiser les protestations politiques, le pouvoir en place annonce, par la voix du Président de la République, qu'il s'apprête à « réviser l'ensemble de l'arsenal législatif sur lequel reposent les règles de l'exercice démocratique et le libre choix des citoyens $»^{4}$. Parallèlement, le pouvoir œuvre à travers la distribution de la rente à contenir la protestation sociale et politique. La réforme menée est une manière de répondre aux revendications de la classe politique et de la société civile en faveur d'un changement pacifique du régime. Cette réforme semble donc être menée sous la pression de l'opinion internationale et nationale. Dans cet "arsenal de réformes", la place de la femme algérienne dans les instances politiques est valorisée. En effet, le Président annonce à cette occasion que le « dépôt et la promulgation de la loi organique relative à la représentation des femmes au sein des assemblées élues interviendront avant les prochaines échéances électorales " (les législatives de mai 2012). Cette loi, dont il sera question ci-dessous, fait de la représentation des femmes dans les assemblées élues un objet de réforme politique et institutionnelle. Elle complète les amendements des lois concernant les partis politiques, les associations, le régime électoral et l'information, toutes adoptés par le Parlement au début de l'année 2012.

5 Cet intérêt pour les droits politiques et civiques des femmes a pris en effet un relief particulier depuis le début des «révolutions arabes». En Algérie, bien que le mouvement de contestation politique et social soit limité, géographiquement et numériquement ${ }^{5}$, la mobilisation des femmes en a été une composante non négligeable. C'est pour le pouvoir algérien l'occasion d'accélérer une réforme amorcée en 2008, lorsque la Constitution a été amendée pour instaurer un nouvel article 31 bis permettant l'augmentation « des chances d'accès des femmes à la représentation dans les assemblées élues ${ }^{6}$. Comme l'attestent les chiffres, la présence des femmes dans les institutions publiques et dans les sphères de prise de décision en Algérie est très faible. 
Trois femmes seulement détiennent des portefeuilles ministériels au gouvernement. Elles n'occupent que 7,7 \% des sièges à l'Assemblée Populaire Nationale de 2007, seules 7 femmes (toutes désignées par le Président de la République) siègent dans le Conseil de la Nation composé de 144 membres $^{5}$, seules trois femmes sur 1451 sont élues maires (suite aux élections de 2007), et une seule femme sur 48 est désignée au poste de wali (préfet).

6 Les députés opposés au projet de loi ont d'emblée reproché au gouvernement d'imposer un quota pour les femmes au Parlement alors que celui-ci ne fait pas de même quant à la désignation aux postes à responsabilités. Lors de ces débats, le député Ahmed Hasnaoui, pourtant affilié au parti du Front de Libération Nationale, membre de la coalition gouvernementale, a demandé au gouvernement d'adopter en contrepartie une autre loi imposant un quota de $30 \%$ des femmes dans le gouvernement, dans les postes de wali (préfet), de chefs de dầîra (sous-préfets), au Conseil de la Nation, et même dans les tribunaux et les conseils juridiques. En effet, la sous-représentation des femmes, qui témoigne des inégalités entre les sexes, ne reflète pas la réalité socio-professionnelle des femmes algériennes. Car la forte présence des femmes dans des secteurs importants comme l'éducation, la santé, l'administration et la justice atteste de leur participation vitale à l'activité sociale et économique. À cela s'ajoute un taux important de scolarisation des filles : dans l'enseignement supérieur, leur présence dépasse $50 \%$ de l'effectif estudiantin, et dans certaines spécialités, comme les lettres et les sciences humaines, ce taux dépasse les $60 \%$.

7 En instaurant dans la Constitution un article qui confirme la volonté de l'État de donner aux femmes une place considérable dans les assemblées élues, le pouvoir tient compte de leur sous-représentation, mais aussi de leur rôle dans la vie sociale et économique. Sont également loués sa participation à la guerre de libération et «ses sacrifices et sa résistance extraordinaire pendant de longues années face à la machine meurtrière et destructive de la décennie noire $»^{7}$. Une telle reconnaissance du rôle des femmes se révèle nécessaire pour obtenir leur soutien pour le processus des réformes, et surtout pour assurer une forte participation des femmes dans les élections législatives et locales organisées en 2012. Dans sa campagne visant à mobiliser les Algériens pour se rendre massivement aux urnes, surtout après l'appel au boycott lancé par quelques associations et partis politiques, le Président Bouteflika a mis l'accent sur la participation des femmes pour réussir ces élections ${ }^{8}$. Il a ainsi incité les responsables des partis et de l'administration à «tenir compte de l'esprit et de la lettre de la Constitution qui vise essentiellement à encourager la femme à participer à la vie politique et élargir sa contribution à l'édification démocratique ».

Dans un contexte particulier, marqué sur le plan national et régional par un fort mouvement de contestation du pouvoir politique et de protestation sociale, la réussite des élections législatives est devenue pour le pouvoir un enjeu majeur afin de rétablir sa crédibilité et consolider sa légitimité politique. Dans une étape qu'il qualifie de " décisive dans l'histoire du pays ", le Président s'adresse à nouveau à ses concitoyens, deux jours avant les élections, pour les persuader d'aller voter. Il appelle ainsi les Algériens, «femmes et hommes, à être à la hauteur des défis qui s'imposent au pays pour affirmer [leur] engagement et [leur] mobilisation collective, et répondre à l'appel de la nation ", c'est-à-dire à voter. Il ne rate pas l'opportunité de souligner que les élections législatives de 2012 se distinguent des précédentes par « une forte présence 
des femmes sur les listes de candidatures, favorisée par l'évolution démocratique dans notre pays".

9 C'est dire combien le discours officiel a fait des élections législatives une bataille pour le renforcement de sa légitimité, et de la forte présence des femmes sur les listes électorales un argument allant dans le sens de la consolidation du principe d'égalité entre les sexes. Ainsi, le renforcement de la représentation des femmes dans les assemblées élues est considéré comme une évolution démocratique, mais une évolution qui s'impose selon la logique, la stratégie et les objectifs propres au pouvoir. Sans aucune concertation avec les mouvements féminins et féministes, ni avec les partis politiques, le gouvernement présenta son projet de loi comme relevant de ses attributs constitutionnels, et émanant de la volonté de réforme menée par le chef de l'État. Les femmes algériennes apparaissent ainsi dans le discours du gouvernement comme un groupe homogène. Les positions et propositions qui avaient été présentées par les femmes et par les associations défendant leurs droits politiques et civiques, à l'occasion du débat (en appelant à un changement réel du statut des femmes dans la sphère politique, sociale et économique $)^{9}$, n'ont pas été prises en compte.

10 Il importe également de souligner que des dynamiques internationales et nationales complexes ont été favorables à une telle loi. Car elle se situe dans la continuité des politiques publiques mises en œuvre par l'État algérien afin de promouvoir le statut social des femmes ${ }^{10}$. Ces politiques répondent, en effet, aux exigences des conventions internationales, notamment la «Convention pour l'élimination de toutes les formes de discrimination à l'égard des femmes ", ratifiée en 1996 par l'Algérie avec des réserves, et le suivi et application de la déclaration et du Programme d'action de Beijing, qui considère que « les femmes, l'exercice du pouvoir et la prise de décision sont parmi les domaines critiques dans les plans et les stratégies ».

\section{Les quotas de femmes : l'avènement d'une loi}

11 En quoi une loi sur les quotas de femmes dans les élections peut-elle contribuer à leur rendre justice ? En quoi peut-elle contribuer au changement politique ?

Le débat sur les quotas de femmes dans les parlements et les assemblées locales n'a cessé ces deux dernières décennies de susciter de nombreuses controverses. Les hommes comme les femmes sont divisés sur la question. Les arguments contre les quotas et en sa faveur se structurent autour de deux idées principales : l'égalité et la représentation.

13 En effet, le pouvoir politique algérien a mis en exergue, en instaurant l'article 31 bis cité ci-dessus, le principe de la représentation. Toutefois, celle-ci ne prend pas la forme d'une "politique des idées », dans le sens d'Anne Phillips ${ }^{11}$, c'est-à-dire d'une présence par laquelle se traduisent effectivement les revendications et les intérêts des femmes, quelles que soient leurs divergences. Car dans le discours officiel, l'accent est surtout mis sur « la présence en nombre » des femmes. Or, la représentation des femmes dans les instances politiques ne peut être réelle et efficace que si les inégalités de fait dont elles sont victimes sont corrigées à travers les politiques et les actions gouvernementales. Dans cet esprit, le mouvement féminin et féministe en Algérie ${ }^{12}$ considère que la situation des femmes algériennes constitue une injustice " par rapport au rôle historique que les femmes algériennes ont joué dans la vie politique du pays ", qu'elle n'est pas en conformité avec les avancées enregistrées en matière de droits 
sociaux et économiques des femmes, et qu'elle crée un sentiment d'exclusion et une contradiction ${ }^{13}$.

14 En 2006, des représentantes de partis politiques, d'associations féminines et d'institutions publiques discutant la question de la représentation égalitaire des femmes dans les institutions politiques et publiques ont revendiqué :

- l'instauration d'un quota de présence (au minimum de $30 \%$ ) de femmes sur les listes des partis politiques et son corollaire, une sanction financière en cas d'infraction ;

- l'obligation de généraliser la formule à toutes les formes de scrutin, y compris les scrutins à caractère local. C'est en effet à ce niveau que s'exerce la vraie démocratie représentative, et les femmes ne doivent pas en être exclues (ce sont les auteurs qui soulignent).

elon les associations féminines algériennes, il est temps de « rendre effectif le principe d'égalité politique et publique entre hommes et femmes ». La responsabilité qui pèse sur le gouvernement et l'obligation juridique qui lui incombe sont celles de faire de la loi sur les quotas des femmes un mécanisme qui achemine vers la consécration du principe d'égalité complète entre les hommes et les femmes. C'est dire que pour les associations algériennes de défense des droits des femmes, le renforcement de la présence féminine dans les assemblées élues ne devrait pas être une fin en soi, mais le commencement de l'application de mesures égalitaires en faveur des femmes, en tenant compte de leurs compétences et de leur forte présence dans la sphère publique.

Ainsi, pour Fadhéla Chitour, féministe, membre du Réseau Wassila qui fédère plusieurs associations de défense des droits des femmes, cette loi « n'a aucun sens tant que les droits des citoyens ne sont pas respectés, tant que la femme n'est pas considérée comme partie intégrante de la vie publique, comme individu à part entière. Quelle valeur peut avoir cette "reconnaissance" et cette "promotion" sans l'abrogation du code de la famille, qui maintient la femme dans une infériorité infamante? $»^{14}$. La présidente de l'association Djzairouna (association des familles des victimes du terrorisme), Mme Cherifa Kheddar, qui est aussi la porte-parole de l'Observatoire des violences faites aux femmes, estime que «le nombre, important ou symbolique, de députées ou de ministres en politique, n'est pas ce qui réglera les discriminations vécues au quotidien par les femmes $»^{15}$.

17 Annoncée officiellement depuis le remaniement de la Constitution de 2008, la représentation des femmes dans la vie politique a été axée sur les assemblées élues seulement ${ }^{16}$. Cependant, en faisant de la représentation des femmes dans ces assemblées un domaine relevant des lois organiques, le pouvoir algérien a décidé de la placer au-dessus des lois ordinaires ${ }^{17}$ : c'est un geste de sa part pour souligner l'importance qu'il accorde au sujet. Il répond ainsi, comme nous l'avons montré plus haut, d'un côté aux revendications des associations de défense des droits des femmes ${ }^{18}$, et de l'autre, aux recommandations et critiques des organisations onusiennes et des $\mathrm{ONG}^{19}$. Mais force est de constater qu'il a fallu attendre pas moins de trois ans pour que cette loi organique soit adoptée. Cela montre au moins la difficile maturation de cette loi, ainsi que la lente prise de conscience de la part du pouvoir politique de l'importance de prendre les mesures nécessaires pour permettre une représentation politique équilibrée des femmes. Notons d'ailleurs que la prise de conscience de cette nécessité n'est pas synonyme d'une conscience de genre qui, selon la définition de Manon Tremblay, «traduit le fait de reconnaître que le sexe (c'est-à-dire le fait d'être une femme ou un homme) modèle d'une façon importante la relation qu'une personne entretient avec la société politique dans laquelle elle évolue $»^{20}$. Enfin, soulignons que si 
l'adoption de l'article 31 bis a été adoptée en 2008, c'est également en raison d'une conjoncture spécifique. Le remaniement constitutionnel de 2008 visait en effet avant tout à permettre au Président de la République de se présenter pour un troisième mandat, et à renforcer ses pouvoirs.

\section{Les étapes du processus d'adoption de la loi}

18 L'adoption de cette loi organique a été particulièrement complexe et a suscité de vifs débats dans le Parlement et dans les partis politiques.

\section{L'avant-projet de la commission}

19 La commission créée en mars 2009 par le Président de la République pour la préparation de l'avant-projet - sous la responsabilité du ministre de la Justice, garde des Sceaux - était composée en majorité de femmes. Les membres de la commission étaient des juges à la Cour suprême et au Conseil d'État, des universitaires (sociologues, politologues, juristes) et des personnalités représentant des ministères et des institutions étatiques ayant trait à la condition des femmes. En attribuant la mission de concrétiser l'article 31 bis de la Constitution au ministère de la Justice, et non pas au ministère de la Condition féminine, qui a pourtant a été le précurseur de la loi sur les quotas, le pouvoir algérien a mis en avant le caractère politique de cette loi en lui donnant une portée importante.

L'avant-projet de loi présenté au gouvernement était composé de 12 articles. Il prévoyait que les listes des candidats dans les assemblées populaires communales et de wilayas, ainsi qu'à l'assemblée populaire nationale, devaient comprendre un nombre de citoyennes égal au moins à $30 \%$ de l'ensemble des candidats inscrits sur la liste. Mais le plus important dans cet article, c'est que les candidats des deux sexes doivent être classés alternativement dans l'ordre de présentation de liste. Cela donne aux femmes la chance d'être élues à un taux qui ne sera pas inférieur à $30 \%$. Le non-respect de ces quotas entraîne le rejet des listes ${ }^{21}$. Loin de se borner à la question des quotas dans les assemblées élues, comme il est prévu dans l'article 31 bis, l'avant-projet apporte des proposition allant jusqu'à exiger des partis politiques que « la composition de l'organe délibérant et de l'organe exécutif du parti politique (...) doit comprendre au moins $30 \%$ de citoyennes choisies parmi les membres du parti», sous peine de se voir refuser l'agrément par le ministère de l'Intérieur.

21 Dans une autre mesure, visant à inciter les partis à présenter le plus grand nombre de femme, le projet de loi a prévu des aides financières spécifiques de l'État selon le nombre de femmes élues. Le même article a été adopté dans la loi organique finale.

\section{L'adoption du projet de loi par le gouvernement}

Le projet de loi organique a été adopté lors du conseil des ministres présidé par Abdelaziz Bouteflika, le 28 août 2011. Il confirme que la proportion de candidates ne pourrait être inférieure au tiers, mais réserve cette condition aux communes de plus de 20000 habitants, dont le nombre est de 792 (sur 1541 communes). Le représentant du gouvernement, ici le ministre de la Justice, justifie cette restriction par la crainte de ne pas trouver de candidates dans les petites communes à cause de l'emprise des 
traditions et des coutumes. En outre, le projet du gouvernement n'oblige pas les listes des partis politiques et des indépendants à classer les deux sexes alternativement, comme le recommandait le projet de la commission. Il confirme cependant la règle du tiers des sièges réservés aux candidates, selon leur classement nominatif sur la liste concernée. Enfin, le projet adopté par le gouvernement souligne que «dans le cas où une liste obtient deux sièges seulement, ces derniers seront répartis entre les deux sexes, selon le classement nominatif des candidats ».

\section{L'adoption de la loi par le Parlement}

Si, au sein du gouvernement, l'adoption de la loi organique est passée avec des modifications qui ne touchent pas au fond du texte, le débat dans les deux chambres du Parlement, et surtout au sein de l'APN, a entraîné de vives controverses. Il révèle de grandes divergences au sein des partis politiques quant à la représentation des femmes. Les débats témoignent, parfois jusqu'à la caricature, de la sensibilité que revêt cette question.

Dans un raisonnement prenant en compte les réserves, voire le refus de certains partis politique d'adopter la loi organique telle qu'elle a été présentée par le gouvernement, la commission des affaires juridiques et administratives et des libertés, instance chargée d'examiner la loi avant sa présentation au Parlement (composée dans sa majorité des députés représentants des partis conservateurs et islamistes), a touché à l'efficience même de la loi. Conscient de la complexité de cette dernière, le rapporteur de la commission appelle les députés à la "sagesse et la rationalité ", à la "responsabilité et la solidarité ", au " calme » et à la " sérénité ". C'est dire à quel point le projet de loi a été l'objet d'âpres critiques dans la classe politique et d'un minutieux examen par le gouvernement, qui rarement, et peut être jamais, n'a vu un projet de loi organique qu'il a présenté susciter tant de différends.

En effet, la commission a préféré un projet de loi qui réduit le quota des femmes dans les assemblées élues au cinquième au lieu du tiers. Encore une fois, la question des coutumes et des traditions a été mise en exergue pour justifier cette régression. Les membres de la commission ont également justifié cela par la difficulté de trouver des femmes candidates. Ont été également convoqués les facteurs religieux, sociaux et culturels qui marquent les rapports sociaux de sexes en Algérie. Ainsi, comme l'écrit le juriste Nouredine Saadi, les femmes algériennes font face à un « obstacle fait de pathos religieux, coutumier ou ancestral, qui oppose une tradition assimilée à soi-même, à l'authenticité, contre l'universel, considéré comme l'autre, l'agresseur ${ }^{22}$.

Malgré la position de la commission juridique, le ministre de la Justice a défendu le principe de l'obligation d'un tiers de femmes dans les listes de candidatures. Dans un discours se voulant ouvert, rigoureux et pédagogique, le représentant du gouvernement a fait l'éloge du rôle de la femme dans la société en défendant le principe des quotas pour femmes, en vigueur dans 87 pays. Le pourcentage de $30 \%$ proposé par le gouvernement a été défendu pour être comparable à celui de la présence des femmes dans la fonction publique. Le projet de loi s'inscrit, selon le représentant du gouvernement, dans le cadre évolutif et moderniste de la société algérienne. Défendant avec opiniâtreté et fermeté son projet, le ministre appelle les députés à assumer leurs responsabilités devant "l'histoire et leur conscience», un appel inaccoutumé dans l'histoire de la législation algérienne de la part d'un membre du gouvernement. 
Après les débats qui ont suivi les interventions des députés et du ministre, les deux parties (gouvernement et députés) sont arrivées à un compromis instaurant les quotas des femmes dans les assemblées élues de la manière suivante :

1. Élections de l'Assemblée Populaire Nationale :

$20 \%$ lorsque le nombre de sièges est égal à 4 sièges,

$30 \%$ lorsque le nombre de sièges est égal ou supérieur à 5 sièges,

$35 \%$ lorsque le nombre de sièges est égal ou supérieur à 14 sièges (trois wilayas sont concernées)

40 \% lorsque le nombre de sièges est égal ou supérieur à 32 sièges (seule la Wilaya d'Alger a autant de sièges)

$50 \%$ pour les sièges de la communauté nationale à l'étranger.

2. Élections des assemblées populaires communales (APC) :

$30 \%$ pour les assemblées populaires communales situées dans les chefs-lieux des dầîra et au sein des communes dont le nombre d'habitants est supérieur à vingt mille (20000) habitants.

3. Élections des assemblées populaires de wilayas (APW) :

$30 \%$ lorsque le nombre de sièges est de $35,39,43$ et 47 sièges.

$35 \%$ lorsque le nombre de sièges est de 51 à 55 sièges.

\section{L'avis du Conseil Constitutionnel}

La priorité est donnée au nombre de femmes sur les listes électorales en premier lieu et en second lieu, à leur positionnement sur ces listes. Le modèle de représentation est donc fondé sur le nombre de sièges de chaque circonscription électorale. Étant donné que les circonscriptions sont pourvues en sièges en fonction du nombre d'habitants, cette loi instaure une sorte de "discrimination territoriale " (notamment en ce qui concerne les APC) justifiée par des arguments sociaux et culturels.

Malgré cette modification, l'essentiel demeure toutefois, pour le gouvernement, de permettre aux femmes d'être présentes dans les assemblées élues. Le Conseil Constitutionnel admet d'ailleurs que cette disposition contestée a été adoptée "pour éviter que les listes électorales dans ces APC ne soient rejetées si elles ne comportent pas un nombre suffisant de femmes en raison de contraintes socio-culturelles ». Le juge parait ainsi en quelque sorte accepter la situation «socio-culturelle», sans inciter le gouvernement à prendre les mesures nécessaires pour y faire face.

Sur saisine du Président de la République, la loi organique a en effet été déférée au Conseil Constitutionnel afin de contrôler sa conformité à la Constitution. Si le juge constitutionnel ne change pas la loi sur le fond, il émet néanmoins un avis qui touche à sa forme, et plus précisément à son intitulé. Il estime en effet que le titre de "loi organique fixant les modalités d'élargissement de la représentation des femmes dans les assemblées élues » constitue " une omission qu'il y a lieu de corriger au libellé et à l'article $1^{\text {er }}$ de la loi organique ", en conformité avec l'article 31 bis de la Constitution, qui parle de "la promotion des droits politiques de la femme en augmentant ses chances d'accès à la représentation dans les assemblées élues ». Finalement, le libellé de la loi organique a été rédigé comme suit: «loi organique fixant les modalités augmentant les chances d'accès de la femme à la représentation dans les assemblées élues ».

31 En évoquant, dans la Constitution et la loi organique, l'augmentation des « chances des femmes », la législation algérienne adhère au principe des quotas non pas dans le sens 
de l'égalité entre les sexes, mais dans celui de l'augmentation méthodique du nombre des femmes élues. La loi en question néglige la répartition équilibrée des hommes et des femmes sur les listes des candidatures aux élections de l'APN, des APC et des APW. Car c'est $46 \%$ du corps électoral qui se voit représenté par un taux qui varie de $20 \%$ à $50 \%{ }^{23}$. Il ne s'agit donc pas de l'application du principe d'égalité, mais d'une mesure politique imposant aux partis politiques de contribuer au renforcement de la représentation des femmes dans les assemblées élues, sans qu'il n'y ait aucune mesure de la part du gouvernement dans les postes qui ressortent de ses propres compétences, sauf en ce qui a trait à la fonction publique ${ }^{24}$. Il ne s'agit pas d'une question de représentativité des femmes dans la vie politique et publique, ni même d'une forme de discrimination positive à l'égard des femmes, mais d'une réponse à l'exigence qui s'impose aux États ayant signé des traités internationaux, notamment la Convention sur l'élimination de toutes les formes de discrimination à l'égard des femmes, ratifiée par l'Algérie en 1996, ainsi que la déclaration de Pékin ${ }^{25}$. La réaction de l'Algées femmes et assurer leur représentation égale à tous les niveaux de responsabilité au sein des institutions nationales et international ${ }^{26}$. Même s'il n'y est pas prévu de sanction contre les pays n'ayant pas respecté leurs engagements, la question de l'égalité des sexes a pris de l'ampleur dans les rapports des institutions onusiennes et des ONG œuvrant pour la défense des droits des femmes et de l'homme. Dans le même temps, l'enjeu électoral pour le pouvoir en place était autre que la présence des femmes dans le Parlement, puisque le renforcement de la présence au Parlement du FLN, soutenu publiquement par le Président de la République, a constitué son principal objectif, dans le but d'assurer la pérennité et la stabilité du régime.

\section{La position des parlementaires face à la question des quotas}

Il convient de remarquer tout d'abord qu'avant la loi organique sur les quotas des femmes dans les assemblées élues, la question de la place des femmes dans la vie politique n'avait guère été discutée par les parlementaires ${ }^{27}$. Au sein des deux chambres du Parlement, aucune proposition de projet n'avait été présentée dans ce sens par les députés. Tout a donc commencé avec l'instauration de l'article 31 bis de la Constitution en 2008. C'est donc la volonté politique qui a primé, grâce notamment à l'autonomie dont dispose l'exécutif dans le choix des moments et des lieux de son intervention. Mais la volonté n'est pas tout, car même si elle émane du constituant ou du législateur, « elle doit être conjuguée à d'autres facteurs, accompagnée d'une mobilisation et d'une sensibilisation des hommes et des femmes en faveur du changement des rapports sociaux de genre $»^{28}$.

Les débats sur ce sujet ont reflété les positions politiques et idéologiques très divergentes des partis politiques, en traduisant les projets de société dont ils sont les porteurs. Le parti des Travailleurs, opposé à toute idée de quotas pour les femmes, bien qu'il ait voté l'amendement de la Constitution introduisant l'article 31 bis, s'est opposé au projet de loi organique. Selon la porte-parole du parti, Mme Louisa Hanoune, «le système du quota de femmes aux assemblées élues peut entraîner des dérives et ne sert pas les intérêts de la femme, qui a arraché ses droits par les armes durant sa participation à la Révolution " ${ }^{29}$. Elle l'a même qualifié "d'escroquerie politique, de scandale et de mascarade $»^{30}$. Toutefois, et contrairement aux autres partis, elle ne 
justifie pas son opposition aux quotas par le poids des coutumes et traditions, mais par l'idée qu'il n'appartiendrait qu'aux partis eux-mêmes de présenter, volontairement, des femmes sur leurs listes électorales. Le parti des Travailleurs se veut d'ailleurs précurseur en ce domaine. Pour les élections législatives de 2007 déjà, il a présenté 17 femmes comme têtes de listes. En 2012, près de la moitié de ses listes ont été conduites par des femmes. Ce parti est celui qui a présenté le plus grand nombre de femmes aux élections législatives et, sur les 31 femmes élues au Parlement, 10 l'ont été sur ses listes. En s'opposant à la loi des quotas, le parti critique les politiques gouvernementales en matière de droits féminins, puisque l'urgence, selon son porte-parole, est de « lever les obstacles devant les femmes dans le code de la famille et dans la précarité ». L'attachement à l'idée d'égalité et le risque d'institutionnaliser les différences entre les sexes sont deux arguments avancés par le parti pour justifier sa position.

Les débats sur les quotas ont pris une orientation à caractère polémique du fait qu'ils touchent à la question du statut des femmes dans la société et dans la vie politique, et également à l'image que se font de la femme les partis politiques et les députés. Trois éléments principaux ont ainsi été mis en avant pour légitimer la position des députés : les traditions et les coutumes de la société, le manque d'expérience et de compétences parmi les femmes, et l'importance d'une application progressive de toute procédure visant à augmenter la présence des femmes dans les assemblées élues. Zohra Drif Bitat, personnalité historique et vice-présidente jusqu'en 2011 du Conseil de la Nation ${ }^{31}$, deuxième chambre du Parlement, a vertement critiqué ces différents arguments. Selon elle, il s'agit de prétextes illusoires: "les femmes instruites sont en force dans les différentes régions du pays : $45 \%$ des magistrats sont des femmes, le taux de réussite des filles à l'examen du baccalauréat était de $65 \%$ en 2010, contre $35 \%$ pour les garçons ». Selon elle, le taux de représentation féminine de $30 \%$ est « le seuil minimum à adopter pour enclencher une dynamique à même de consacrer le principe de parité $»^{32}$.

Par ailleurs, la loi sur les quotas posait un « problème » relatif au communautarisme.

Les représentants de la communauté mozabite implantée dans la wilaya de Ghardaïa (au sud du pays), connue pour sa rigueur pour tout ce qui a trait aux traditions et à la religion, se sont manifestés contre la loi. Au nom du Conseil des Notables qui représente la haute référence ibadite de l'Algérie ${ }^{33}$, la loi est contestée parce qu'elle n'est pas conforme aux traditions d'un pays dont « la plupart des régions demeurent conservatrices et considèrent que le rôle des femmes se réduit à la sphère familiale pour jouer le rôle d'épouse et de mère... » En imposant des quotas pour les femmes, cette loi est jugée "extravagante et discriminatoire envers les hommes $»^{34}$. Mais la position du Conseil des Notables n'a pas fait l'unanimité, et des voix discordantes sont allées jusqu'à contester sa représentativité et sa crédibilité ${ }^{35}$.

Ces débats révèlent également des postures politiques et sociales relevant d'un système de domination. Un député issu du Mouvement pour la Société de la Paix (de tendance islamiste), a ainsi déclaré: "Nous devons être réalistes et objectifs: il s'agit d'incorporer les femmes dans des domaines pour lesquels elles ne sont pas prêtes et où, de plein gré, elles ne veulent pas être impliquées $»^{36}$ (Kamel Guergouri, MSP). D'autres se sont exprimés contre la loi avec virulence, n'hésitant pas à mobiliser un discours ouvertement machiste et patriarcal, tels ce député déclarant : « Mais où est l'homme en Algérie? A-t-il disparu? Est-il temps d'adopter une loi organique qui féminise l'homme? Telle est la réalité. Ce projet de loi va créer une discorde (fitna) entre les 
hommes et les femmes... La femme dispose déjà de tous ses droits dans la société. Ils sont à $100 \%$. Si vous [le gouvernement] lui ajoutez les $20 \%$ que vous ôtez à l'homme, ce taux devient alors $120 \%$... Serions-nous plus juste que Dieu? La femme a-t-elle besoin d'être membre dans les assemblées élues ou d'être députée? La femme algérienne, digne, noble et respectueuse, a besoin des choses plus intéressantes que cela...» (Mestfai Ben Attallah, FLN). Cette position reflète une vision stéréotypée et subjective du rôle de la femme algérienne dans la société, présume que la situation des femmes est idéale, et justifie même cette situation par le fait qu'elle serait la volonté de Dieu. Pour les tenants de cette position, la question des quotas est un « faux débat ", puisqu'il n'y a pas de problème lié à la condition des femmes.

Toutefois, d'autres on mis en avant, dans les débats parlementaires, la question de l'émancipation sociale et culturelle de la femme. Certains députés considèrent que le problème des femmes en Algérie est en premier lieu social, et que les efforts doivent se porter sur cet aspect :

Le système des quotas obligatoires, tel qu'il a été adopté par certains pays diffère selon les caractéristiques culturelles propres à chaque nation. Il ne peut en aucun cas permettre aux compétences féminines de progresser, puisque la priorité sera donnée à l'appartenance sexuelle au lieu de la compétence. Cela va contribuer à prolonger la situation actuelle, et à accumuler les problèmes de mauvaise gestion dont souffrent plusieurs assemblées élues... (Mohamed Mahmoudi, MSP)

Le même discours est repris par des députés qui ne voient pas l'intérêt d'une telle loi, même parmi ceux appartenant à l'Alliance présidentielle :

Quel avantage une telle loi peut-elle apporter à la femme dans l'Algérie profonde? Que peut-elle changer dans le quotidien des millions de femmes malheureuses, ou dans leurs foyers? (...) Les femmes aspirent à une vie digne dans laquelle l'enseignement et la protection sociale seront assurés, ainsi qu'à un logement décent et du travail pour leurs enfants. Elles aspirent à une vie respectable qui les éloigne de la pauvreté et de la misère. Décorer le Parlement avec quelques femmes députées ne les sert en rien (Mahdi Kassimi Hassani, RND).

Quant aux députés de l'opposition, certains d'entre eux doutent des intentions du pouvoir et estiment que la position de la commission juridique, qui ne s'oppose que rarement aux projets de lois présentés par le gouvernement, est une ruse employée par ce dernier pour réduire la représentation des femmes du tiers au cinquième et édulcorer ainsi les dispositions de la loi sur les quotas :

Le gouvernement visait dès le début un pourcentage de $20 \%$ et comptait sur les députés pour cela, tout en se présentant comme étant plus progressiste que les élus du peuple. C'est une ruse qui a pour objectif d'enjoliver le pouvoir, lequel a pratiqué une politique discriminatoire à l'égard des femmes (Tareq Mira, RCD).

De la même manière, un député du parti islamiste Nahda considère le projet de loi comme une tentative du pouvoir de se dérober aux réformes réelles et de dissimuler l'échec de ses politiques de développement :

Les députés de l'Alliance [qui constituent la majorité] ont refusé récemment une proposition du parti Nahda visant à accorder une allocation aux femmes au foyer, et tentent aujourd'hui de gagner de l'argent à travers ce projet, par le biais des candidatures féminines. C'est une corruption légitimée (Mohamed Ahdibi, Nahda).

41 Pour l'opposition, c'est donc l'occasion de régler ses comptes avec le gouvernement. La loi sur les quotas des femmes est considérée comme une fuite en avant de ce dernier, qui a échoué à tenir ses promesses et à réaliser le programme présidentiel de développement. Les réformes engagées n'ont pas constitué une avancée dans le 
processus de changement et de démocratisation. Le programme présidentiel de développement, quant à lui, n'a pas donné les résultats escomptés. Le débat parlementaire sur les quotas des femmes été l'occasion de mettre l'accent sur d'autres problèmes liés à la situation des femmes algériennes, comme les conditions de travail, les salaires, la désignation des femmes à des postes à responsabilités, etc.

Mais la parité n'a guère été revendiquée dans tous ces débats, qui se sont concentrés sur les obstacles liés à la candidature des femmes et à la difficulté de les mobiliser dans les partis politiques et dans la campagne électorale, ou sur la condition des femmes en général. La loi n'a pas inscrit l'obligation de l'alternance homme et femme (ou femme et homme) sur les listes de candidatures. Dans un régime électoral proportionnel, c'est surtout cette mesure qui permettrait de réaliser la parité dans la représentation des femmes. Le principe de citoyenneté, comme élément contribuant non pas à l'augmentation de la présence des femmes mais à leur émancipation dans la sphère politique, a également été ignoré. La loi organique est loin de contenir une conception de la citoyenneté, pleine et entière, en tant que dynamique consolidant le principe d'égalité, selon lequel les femmes participent à la vie de la société en tant que membres à part entière. C'est en effet à cette condition que peut s'exercer la citoyenneté pratique, "notamment à travers la représentation et la participation politiques qui fournissent à l'individu la capacité d'avoir de l'influence sur l'espace public ${ }^{37}$.

En outre - en dépit de la volonté de quelques partis politiques, qui, bon gré mal gré, se trouvent dans l'obligation de respecter la loi après son adoption et de présenter des femmes sur leurs listes électorales -, trouver des candidates est une tâche difficile pour eux à cause de la très faible proportion de femmes en leur sein. L'on comprend mieux ainsi les raisons pour lesquelles les députés et les chefs de partis politiques ont été amenés à s'opposer à la loi organique. Considérée comme problématique pour les partis politiques, l'imposition d'un quota féminin représente le côté ambivalent de cette loi. Moussa Touati, chef du Front National Algérien, considère que cette loi obligera les partis «à proposer la candidature de femmes n'ayant aucune relation avec les programmes et les idées des formations politiques. Ce fait induira un recul du niveau politique au sein du prochain Parlement, car les partis ne disposant pas de compétences féminines se retrouveront obligés de proposer des candidates ordinaires $»^{38}$. Faisant le lien entre la date de la présentation de la loi et les émeutes qui ont secoué le pays en 2011 pour des raisons sociales et économiques notamment, il estime que les autorités "veulent faire diversion avec cette histoire de quota dans le but de détourner l'attention des véritables problèmes qui se posent à la société $\aleph^{39}$.

Les contraintes des partis ont ainsi été prises en compte dans l'élaboration des listes de candidatures, puis dans le déroulement de la campagne électorale. Certaines affiches ont été posées sans même les photos des femmes, notamment dans les villes intérieurs et du Sud, mais également dans quelques villes anciennes comme Tlemcen. Même des partis de tendance laïque se sont abstenus d'afficher les photos de leurs candidates, par mesure de compromis avec les traditions locales, comme par exemple le FFS dans la wilaya de Ghardaia.

Ces phénomènes sont à mettre en relation avec le fait que l'enjeu électoral en Algérie est soumis à un jeu social dans lequel «agents sociaux, appareils partisans, intermédiaires, instances formelles et informelles, pouvoirs local et central» interfèrent dans "l'offre de la représentation parlementaire $»^{40}$. Ce jeu commence avec l'ouverture des candidatures. Les femmes algériennes subissent donc les règles 
imposées par le «système " électoral non institutionnel, mais social, culturel et économique.

\section{Après la loi : un premier bilan}

Durant toutes les étapes des élections, les femmes - qui représentent plus de $45,5 \% \mathrm{du}$ corps électoral et la moitié de la population - ont été très courtisées par les partis politiques en tant que candidates, mais également comme électrices. Malgré les inquiétudes qui ont accompagné les débats concernant la campagne électorale des législatives de 2012, la présence des femmes dans ces élections a été significative. Pas moins de 7700 femmes se sont portées candidates, soit un taux de candidature de $30 \%$. Leur forte présence dans des secteurs comme l'éducation, la santé et la justice a permis aux partis politiques de trouver des candidates potentielles.

Cette présence féminine a par ailleurs entraîné un rajeunissement des candidatures : plus de $62 \%$ des candidates ont moins de 40 ans, alors que ce taux est de $44 \%$ seulement chez les hommes. La très faible présence des femmes dans les rangs des partis politiques a en outre entraîné de nombreux parachutages politiques de candidates, fait marquant de ces élections. De ce fait, des critères comme le niveau scolaire, la fonction, la popularité et l'origine des candidates ont primé sur les convictions politiques et l'appartenance partisane. En effet, l'activité des femmes algériennes constitue, selon une grande enquête sur l'intégration socio-économique féminine, un facteur favorable pour leur engagement dans l'espace politique et associatif et pour l'exercice de leur droit de vote et de se porter candidates ${ }^{41}$. Toutefois, « au moment de la confection des listes de candidatures, la désignation de femmes a tendance à ressembler plus à un artifice qu'à un choix réfléchi en faveur de leur promotion $\star^{42}$. Cela correspond, toutes propositions gardées, à la critique formulée par Janine Mossuz-Lavau à l'encontre de "la politique de présence", qui s'exprime à travers les lois des quotas et de la parité : «Comme si on allait choisir les candidats hommes parmi les militants des partis politiques et les candidates femmes dans une sorte de no man's land, hors des organisations, hors des formations politiques ${ }^{43}$.

Compte tenu des résultats de ces élections, les 146 sièges obtenus par les femmes, soit $31,6 \%$ du total, marquent une étape décisive dans leur histoire sociale et politique. L'importance de ces résultats nous amène à poser la question du rôle des femmes dans le futur Parlement. Comment peuvent-elles s'imposer dans une assemblée qui, depuis l'indépendance, a été un champ de législation réservé aux hommes? Quelle forme prendra la représentation féminine? Cette augmentation de la représentation parlementaire des femmes aura-t-elle des répercussion dans la vie sociale, économique et politique? Le tiers des sièges obtenu par les femmes algériennes constitue ce que Drude Dahlerup appelle « le seuil critique », à partir duquel une minorité est en mesure d'influencer le travail parlementaire ${ }^{44}$. Les intérêts spécifiques des femmes en seront-ils mieux défendus?

Si le contexte dans lequel la loi sur les quotas a pris place est celui des réformes politiques, il importe de situer les changements qui en résultent dans la même perspective. Or, si les réformes et le changement ont été revendiqués par le bas, la loi des quotas a été imposée par le haut. À l'inverse des débats sur les quotas et la parité dans les pays européens (en France notamment), qui ont été marqués par une forte implication des mouvements féministes et de la classe intellectuelle ${ }^{45}$, le cas de l'Algérie 
s'est limité à des controverses politiques dans lesquelles les partis défendaient non seulement leur représentation au Parlement, mais aussi leur place dans la vie politique de l'après « Printemps arabe ». Le résultat obtenu - qui n'a été enregistré dans aucun pays arabe, y compris ceux qui ont connu des révolutions renversant les régimes en place comme la Tunisie, l'Égypte ou la Lybie - marque une autre «exception algérienne ». Toutefois, l'action du gouvernement algérien, agissant pour les femmes sans les femmes, est loin d'une forme de féminisme d'État ${ }^{46}$, comme c'était le cas en Tunisie sous le règne de Bourguiba puis de Ben $\mathrm{Ali}^{47}$ (le féminisme d'État a été problématisé par des auteurs comme Cynthia Nelson, dans sa biographie de Doria Shafik, par Baudouin Dupret et Nathalie Bernard-Maugiron, par Islah Jad, etc.), car ici, la représentation des femmes n'est pas pensée à partir du principe de l'égalité entre les sexes.

L'avis du Conseil Constitutionnel mentionné ci-dessus, qui dénomme la loi des quotas «loi organique fixant les modalités augmentant les chances d'accès de la femme à la représentation dans les assemblées élues » résume le mieux, semble-t-il, les objectifs du pouvoir politique algérien. Augmenter les chances d'accès des femmes à la représentation dans les assemblées élues, est la manière par laquelle le pouvoir répond aux pressions internationales et aux revendications des mouvements féminins et féministes. Il s'inscrit ainsi dans le cadre de "l'espace de la cause des femmes" qui, selon la définition de Laure Bereni, se constitue entre lesdits mouvements, le "pôle partisan " (commissions femmes des partis politiques), et le pôle "institutionnel» (instances chargées de défendre les femmes à l'intérieur de l'État) ${ }^{48}$. Par ailleurs, le pouvoir se montre attentif aux recommandations et exigences des instances internationales concernant la cause des femmes.

51 Cependant, le renforcement de la représentation des femmes dans le champ politique s'est vu limité aux assemblées élues sans favoriser l'accès des femmes algériennes à l'ensemble de la vie politique ${ }^{49}$. La loi organique n'a prévu aucune formule pour l'amélioration progressive de la place des femmes au Parlement en vue d'atteindre la parité. Elle ne prévoit pas non plus de dispositions transitoires différenciant la première application des futures échéances électorales. Or, les quotas doivent être, comme le préconise l'Union Interparlementaire " une mesure palliative temporaire, destinée à favoriser l'émergence d'une nouvelle culture aboutissant à la présence équilibrée de femmes et d'hommes tant au Parlement qu'au sein des instances dirigeantes des partis politiques». Mais au lieu de la parité qui consacre la présence équilibrée, c'est le principe de l'équité introduit par le ministre et les parlementaires qui constitue le fondement de la loi. Même le concept d'égalité, tel que contenu dans la Constitution algérienne, ne traduit, selon certaines analyses, que l'idée d'une égalité formelle : «En d'autres termes, alors que le principe d'égalité correspond à une justice arithmétique (commutative, absolue), traitant tous les individus comme schématiquement égaux, le concept d'équité correspond à une notion de justice proportionnelle (distributive). Ce concept définit un traitement différent selon les personnes ou les groupes $»^{50}$.

52 Enfin, notons que le gouvernement n'a pas pris de mesures complémentaires visant à donner «plus de chances» aux femmes d'être présentes dans la vie politique. En témoigne la composition du nouveau gouvernement issu de ces élections : sur 36 membres, le nombre de femmes est resté le même que dans celui du gouvernement précédent, soit trois femmes ministres (dont une ministre déléguée). Sur les douze 
commissions parlementaires, une seule est présidée par une femme (éducation, enseignement supérieur, recherche scientifique et affaire religieuses). Seul un groupe parlementaire sur les six que compte l'APN est présidé par une femme, en l'occurrence celui du parti des Travailleurs.

La question qui se pose aujourd'hui est celle des conséquences de cette politique des quotas au Parlement sur les droits des femmes (sur l'égalité de genre notamment) et sur le processus de réformes politiques. Les femmes députées peuvent-elles agir, pour paraphraser Simone de Beauvoir, comme des « sujets souverains » et autonomes plutôt que comme un « sexe représenté »?

\section{BIBLIOGRAPHIE}

BEN ACHOURBENGHABRIT-REMAOUN S., 2001, « Féminisme d'État : figure ou défiguration du féminisme? ", Mélanges Mohamed Charfi, Tunis, Centre de Publication Universitaire, p. 413. BENGHABRIT-REMAOUN N. (dir), 2006, Femmes et intégration socioéconomique, étude réalisée par le CRASC pour le compte du ministère délégué chargé de la Famille et de la Condition féminine. BENGHABRIT-REMAOUN N., 2012, "Femmes en politique : une minorité en émergence ", in REMAOUN Hassan (dir), L'Algérie aujourd'hui. Approches sur l'exercice de la citoyenneté, Oran, Éditions du CRASC, p. 135-164.

BERENI L., 2007, « Des quotas à la parité : féminisme d'État et représentation politique (1974-2007) ", Genèses, n 67, p. 5-23.

- 2009, «Quand la mise à l'agenda ravive les mobilisations féministes. L'espace de la cause des femmes et la parité politique (1997-2000) », Revue française de science politique, vol. 59, p. 301-323.

CHENA S., 2011, « L'Algérie dans le "Printemps arabe" entre espoirs, initiatives et blocages ", Confluences Méditerranée, vol. 77, n², p. 105-118.

CIDDEF, 2006, Mémorandum pour une représentation égalitaire des femmes dans les institutions politiques et publiques.

DAHLERUP D., 1988, "From a Small to a Large Minority: Women in Scandinavian Politics", Scandinavian Political Studies, vol. 11, n 4, p. 275-298.

DRIS AÏT HAMADOUCHE L. \& DRIS C., 2012, « De la résilience des régimes autoritaires : la complexité algérienne ", L'Année du Maghreb, vol. VIII, p. 279-301.

GRABA G. \& HADDAB Z., 2007, «Femmes-objets ou femmes-sujets : les enjeux du Code de la famille en Algérie », in sow SIDIBÉ A. et al. (dir.), Genre, inégalités et religion, Paris, Éditions des Archives Contemporaines - AUF, p. 37-48.

HACHEMAOUI M., 2003, « La représentation politique en Algérie entre médiation clientélaire et prédation (1997-2002) », Revue française de science politique, vol. 53, n 1, p. 35-72.

HARTANI A. K., 2003, « Femmes et représentation politique en Algérie : Virtualités constitutionnelles, solutions normatives possibles », CIDDEF, http://www.ciddef-dz.com/pdf/ 
autres-publications/article2-femmes-et-representation-politique-en-algerie.pdf, consulté le 23 janvier 2014.

LALAMI F., 2012, Les Algériennes contre le code de la famille, La lutte pour l'égalité, Paris, Presses de Sciences Po.

LOVENDUSKI J. et al. (ed.), 2005, State Feminism and Political Representation, Cambridge, Cambridge University Press.

MARQUES-PEREIRA B., 1999, «Quotas ou parité : Enjeux et argumentation », Recherches féministes, vol. $12, \mathrm{n}^{\circ} 1$, p. 103-121.

MARTIN J. (dir.), 1998, La parité : enjeux et mise en œuvre, Toulouse, Presses Universitaires du Mirail. mossuz-LAVAu J., 1998, Femmes/Hommes. Pour la parité, Paris, Presses de Science Po.

PHILliPs A., 1995, The Politics of Presence, Oxford, Clarendon Press.

RÉSEAU EURO-MÉDITERRANÉEN DES DROITS DE L'HOMME, 2012, « Réformes politiques » ou verrouillage supplémentaire de la société civile et du champ politique ? Une analyse critique, Copenhague.

SAADI N., 1991, La Femme et la loi en Algérie, Alger, Bouchene.

SAÏ F. Z., 2012, « Femmes africaines et politique : éléments pour une approche comparative », in BENGHABRIT-REMAOUN N. \& BENZENINE B. (dir.), Les femmes africaines à l'épreuve du développement, Oran, Éditions du CRASC, p. 99-132.

TREMBLAY M., 1996, « Conscience de genre et représentation politique des femmes », Politique et Sociétés, $\mathrm{n}^{\circ} 29$, p. 93-137.

\section{NOTES}

*. L'auteur remercie les évaluateurs anonymes de la première version de cet article pour leurs lectures attentives et leurs remarques et suggestions.

1. La deuxième Chambre algérienne, le Conseil de la Nation, n'est pas concernée par la Loi organique. Le ministre de l'Intérieur argue que l'élection des membres du Conseil de la Nation par scrutin indirect ne permet pas la mise en place de mécanismes semblables à l'élection des membres de l'APN, des assemblées populaires communales (APC) et de wilayas (APW).

2. Selon les données de l'Union Interparlementaire publiées le $1^{\text {er }}$ février 2013.

3. Louisa Dris Aït Hamadouche et Chérif Dris, «De la résilience des régimes autoritaires : la complexité algérienne ", L’Année du Maghreb, vol. VIII, 2012, p. 280.

4. Discours du Président à la nation, 15 avril 2011.

5. Voir sur ce sujet Salim Chena, «L'Algérie dans le "Printemps arabe" entre espoirs, initiatives et blocages ", Confluences Méditerranée, vol. 77, n 2, 2011.

6. Il convient de rappeler qu'une réforme des codes de la famille et de la nationalité, qui ont représenté des avancées pour les droits des femmes en Algérie, avait précédé cet amendement constitutionnel. Cf. Ghania Graba et Zoubida Haddab, « Femmes-objets ou femmes-sujets : les enjeux du Code de la famille en Algérie ", in Amsatou Sow Sidibe et al. (dir.), Genre, inégalités et religion, Paris, Éditions des Archives Contemporaines - AUF, 2007. 
5. Selon l'article 131 de la Constitution, «Les membres du Conseil de la Nation sont élus pour les deux tiers au suffrage indirect et secret parmi et par les membres des assemblées populaires communales et des assemblées populaires de wilaya. Un tiers des membres du Conseil de la Nation est désigné par le Président de la République parmi les personnalités et compétences nationales dans les domaines scientifique, culturel, professionnel, économique et social ».

7. Discours du Président Bouteflika, 23 février 2011.

8. Le taux officiel de participation était de $43 \%$ avec 1704047 bulletins nuls sur un nombre de votants de 9339026 .

9. Voir sur ce sujet Feriel Lalami, Les Algériennes contre le code de la famille, La lutte pour l'égalité, Paris, Presses de Sciences Po, 2012.

10. Comme l'adoption de la «Stratégie nationale pour la promotion et l'intégration de la femme» en 2008, le «Plan national d'intégration des femmes", et la «Stratégie nationale de lutte contre la violence à l'égard des femmes ».

11. Anne Phillips, The Politics of Presence, Oxford, Clarendon Press, 1995.

12. Il est représenté notamment par l'Association des Femmes Cadres Algériennes (AFCARE), le Rassemblement contre la hogra (l'oppression) des femmes algériennes (RACHDA), l'Association des Femmes Algériennes pour le Développement (AFAD) et le Centre d'Information et de Documentation sur les Droits de l'Enfant et de la Femme (CIDDEF).

13. CIDDEF, Mémorandum pour une représentation égalitaire des femmes dans les institutions politiques et publiques, 2006, p. 4.

14. Cité dans El Watan, 17 octobre 2011.

15. Idem.

16. Les membres des deux chambres se sont réunis comme le prévoit la Constitution pour voter les amendements. 500 voix étaient pour, 21 (celles du parti de l'opposition $\mathrm{RCD})$ contre et 8 abstentions.

17. La loi organique est adoptée à la majorité absolue des députés et à la majorité des trois quarts des membres du Conseil de la Nation (la chambre haute). Elle est soumise à un contrôle de conformité par le Conseil Constitutionnel avant sa promulgation (Art. 123 de la Constitution).

18. C'est ce qu'admet le gouvernement algérien dans le troisième et le quatrième rapports périodiques de l'Algérie sur la mise en œuvre de la convention sur l'élimination de toutes les formes de discrimination à l'égard des femmes (mai 2009 et mars 2012).

19. À titre d'exemple, Mme Rashida Manjoo, rapporteuse spéciale des Nations-Unies sur la violence contre les femmes, ses causes et ses conséquences, déclare suite à sa visite en Algérie en octobre 2010, à propos des mesures prises par la gouvernement algérien pour la promotion des droits des femmes, que : «En dépit de ces avancées, des progrès restent à faire, notamment dans les domaines de l'interprétation et de l'application ». Elle ajoute : «Au cours de mes rencontres avec des victimes, des témoignages indiquent plusieurs tentatives visant à contourner l'esprit de la loi, donnant lieu à d'autres discriminations et injustices ». HTTP://WWW.UN-ALGERIA.ORG/EVENEMENT/RAPPORTEUSE2010/ COMMUNIQUE.PDF 
20. Manon Tremblay, "Conscience de genre et représentation politique des femmes", Politique et Sociétés, n² 29, 1996, p. 99.

21. Ce rejet a déjà été décidé par la loi.

22. Nouredine Saadi, La Femme et la loi en Algérie, Alger, Bouchene, 1991, p. 29.

23. Les $50 \%$ ne concernent que les sièges de la communauté nationale à l'étranger, qui sont au nombre de 8 seulement (sur 462).

24. Il faut rappeler que la proportion de femmes dans la fonction publique est de $31 \%$.

25. «Renforcer le rôle des femmes et assurer leur représentation égale à tous les niveaux de responsabilité au sein des institutions nationales et internationales susceptibles de définir ou d'influencer les politiques relatives au maintien de la paix, à la diplomatie préventive et aux activités connexes... »

26. Il s'agit de la session qui a eu lieu entre le 17 janvier et le 4 février 2011, durant laquelle 37 questions ont été posées au gouvernement algérien dont quatre concernent la participation politique et la participation à la vie publique des femmes.

27. L'APN a organisé, le 3 mars 2010, une journée parlementaire incluant plusieurs thèmes ayant trait aux rôles des femmes dans les assemblées élues et à leurs perspectives futures à la lumière des changements qu'a connus l'Algérie, afin d'améliorer la place et le rôle de la femme dans la promotion des institutions nationales.

28. Fatima Zohra Saï, «Femmes africaines et politique : éléments pour une approche comparative ", in Nouria Benghabrit-Remaoun et Belkacem Benzenine (dir.), Les femmes africaines à l'épreuve du développement, Oran, Éditions du CRASC, 2012, p. 114.

29. La Tribune, 7 juillet 2012.

30. L'Expression, 27 novembre 2011.

31. Madame Zohra Drif Bitat est une figure du militantisme féminin algérien. Elle était parmi les femmes «héroönes» de la guerre de libération algérienne. Après l'indépendance, elle devient avocate, puis désignée comme membre du Conseil de la Nation (parmi le tiers présidentiel).

32. El Watan, 25 novembre 2011.

33. Ce Conseil constitue une autorité civile, sociale et politique. Il est composé des personnalités notables, des représentants des associations actives, des élus locaux et des députés de la communauté.

34. El Khabar, 10 octobre 2011.

35. C'est la position par exemple de M. Kamel Eddine Fekhar, militant pour la défense des droits de l'homme, élu local et ancien membre du Front des Forces Socialistes (parti d'opposition depuis 1963). Selon une déclaration de M. Fekhar « le Conseil des Notables du M'zab n'est pas le tuteur des citoyens de Ghardaïa, il est par contre l'œil et l'oreille du pouvoir central dans la région, sans réel ancrage. Pourquoi ce Conseil ne s'exprimet-il jamais quand les citoyens subissent les dépassements des autorités? (El Watan, 25 octobre 2012).

36. Toutes les citations des députés sont extraites du journal officiel des débats de l'APN, n 249-250-251, novembre 2011.

37. Bérengère Marques-Pereira, "Quotas ou parité : Enjeux et argumentation ", Recherches féministes, vol. 12, n 1, 1999 , p. 104. 
38. El Moudjahid, $1^{\mathrm{er}}$ Mars 2012, p. 4.

39. Le Midi Libre, 8 septembre 2011.

40. Mohammed Hachemaoui, «La représentation politique en Algérie entre médiation clientélaire et prédation (1997-2002) », Revue française de science politique, vol. 53, $\mathrm{n}^{\circ} 1$, 2003 , p. 36.

41. Nouria Benghabrit-Remaoun (dir), Femmes et intégration socioéconomique, étude réalisée par le Centre de recherche en anthropologie sociale et culturelle (CRASC) pour le compte du ministère délégué chargé de la Famille et de la Condition féminine, 2006.

42. Nouria Benghabrit-Remaoun, «Femmes en politique : une minorité en émergence », in Hassan Remaoun (dir), L'Algérie aujourd'hui. Approches sur l'exercice de la citoyenneté, Oran, Éditions du CRASC, 2012, p. 156.

43. Janine Mossuz-Lavau, Femmes/Hommes. Pour la parité, Paris, Presses de Science Po, 1998, p. 91.

44. Drude Dahlerup, "From a Small to a Large Minority: Women in Scandinavian Politics", Scandinavian Political Studies, vol. 11, $\mathrm{n}^{\circ} 4$.

45. Jacqueline Martin (dir.), La parité: enjeux et mise en cuvre, Toulouse, Presses Universitaires du Mirail ; Laure Bereni, « Des quotas à la parité : féminisme d'État et représentation politique (1974-2007)», Genèses, n 67, 2007.

46. Le féminisme d'État constitue une réponse aux revendications des mouvements féministes à travers des mécanismes et des institutions publiques qui ont pour but de rendre concrète et réelle l'égalité entre les hommes et les femmes sur tous les plans de la vie sociale, politique et économique, tout en œuvrant à l'amélioration de la condition des femmes. Voir Joni Lovenduski et al. (ed.), State Feminism and Political Representation, Cambridge, Cambridge University Press, 2005.

47. Sana Ben Achour , «Féminisme d'État: figure ou défiguration du féminisme?", Mélanges Mohamed Charfi, Tunis, Centre de Publication Universitaire, 2001.

48. Laure Bereni, "Quand la mise à l'agenda ravive les mobilisations féministes. L'espace de la cause des femmes et la parité politique (1997-2000) », Revue française de science politique, vol. 59, 2009, p. 302.

49. Réseau Euro-Méditerranéen des droits de l'Homme, "Réformes politiques" ou verrouillage supplémentaire de la société civile et du champ politique? Une analyse critique, Copenhague, 2012, p. 39.

50. Amine Khaled Hartani, « Femmes et représentation politique en Algérie : Virtualités constitutionnelles, solutions normatives possibles », CIDDEF, HTTP://WWW.CIDDEF-DZ.COM/ PDF/AUTRES-PUBLICATIONS/ARTICLE2-FEMMES-ET-REPRESENTATION-POLITIQUE-EN-ALGERIE.PDF, 2003, consulté le 23 janvier 2014. 


\section{RÉSUMÉS}

La loi des quotas pour les femmes, dite «loi organique fixant les modalités augmentant les chances d'accès de la femme à la représentation dans les assemblées élues ", a été adoptée par le Parlement algérien en 2012. Le pouvoir l'a présentée comme faisant partie du processus des réformes politiques. Toutefois, cette loi était attendue depuis l'amendement de la Constitution en 2008. Ce papier tente en premier lieu de mettre l'accent sur le débat politique et parlementaire sur la loi organique. En second lieu, il est question d'esquisser un premier bilan de cette loi, permettant, malgré toutes les critiques des politiques et des féministes, de classer l'Algérie au premier rang des pays arabes en matière de représentation féminine au Parlement.

The so-called "Organic law defining the modalities to raise the woman chances to gain access to the representation inside elected assemblies", or law for a feminine quota, has been voted by the Algerian Parliament in 2012. The executive power presented this law as a part of the political reform process, even if it took four years to prepare it after the 2008 Constitutional reform. This articles tries to highlight the political and parliamentary debate about this organic law. Then, it examines the first outcomes of this law, which - despite all the critics formulated by politicians and feminists - allows Algeria to rank first amongst the Arab countries on the matter of feminine representation inside Parliament.

\section{INDEX}

Keywords : Quotas, Political representation, Reform, Equality, Citizenship

Mots-clés : quotas, représentation politique, réforme, égalité, citoyenneté

\section{AUTEUR}

\section{BELKACEM BENZENINE}

Belkacem Benzenine est chercheur au Centre national de recherche en anthropologie sociale et culturelle (Oran, Algérie). Il travaille sur la place des femmes dans la vie politique dans les pays arabes. Il a codirigé (avec Amar Mohand Amer) Le Maghreb et l'indépendance de l'Algérie (Karthala, 2012).

Belkacem Benzenine is a researcher at the National Research Center in Social and Cultural Anthropology (Oran, Algeria). He is studying the place of women in the political life of the Arab countries. He co-edited (with Amar Mohand Amer) Le Maghreb et l'indépendance de l'Algérie (Karthala, 2012). 